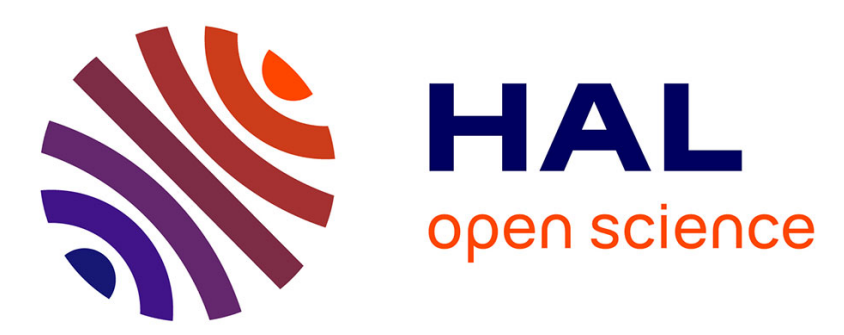

\title{
IDENTIFICATION ET DOSAGE DES ACIDES GRAS LIBRES DU FROMAGE A L'AIDE DE LA CHROMATOGRAPHIE LIQUIDE-LIQUIDE
}

S. Sandev, M. Baltadjieva

\section{- To cite this version:}

S. Sandev, M. Baltadjieva. IDENTIFICATION ET DOSAGE DES ACIDES GRAS LIBRES DU FROMAGE A L'AIDE DE LA CHROMATOGRAPHIE LIQUIDE-LIQUIDE. Le Lait, 1968, 48 (473_474), pp.141-144. hal-00928455

\section{HAL Id: hal-00928455 \\ https://hal.science/hal-00928455}

Submitted on 1 Jan 1968

HAL is a multi-disciplinary open access archive for the deposit and dissemination of scientific research documents, whether they are published or not. The documents may come from teaching and research institutions in France or abroad, or from public or private research centers.
L'archive ouverte pluridisciplinaire HAL, est destinée au dépôt et à la diffusion de documents scientifiques de niveau recherche, publiés ou non, émanant des établissements d'enseignement et de recherche français ou étrangers, des laboratoires publics ou privés. 


\title{
IDENTIFICATION ET DOSAGE DES ACIDES GRAS LIBRES DU FROMAGE A L'AIDE DE LA CHROMATOGRAPHIE LIQUIDE-LIQUIDE
}

\author{
par \\ S. SANDEV et M. BALTADJIEVA \\ (Institut supérieur de technologie des Industries alimentaires, \\ Plovdiv, Bulgarie)
}

L'arôme et les qualités gustatives du fromage sont dus essentiellement à la présence d'acides gras à chaîne courte dont la teneur dépend des processus de fermentation qui ont lieu au cours de la maturation du fromage (Day et Libbey, 1964).

Dans le présent travail nous décrivons une méthode d'identification et de dosage des acides gras libres du fromage. Cette méthode est appliquée à l'étude des acides gras libres du fromage "Kephalotiri ". Ce fromage à pâte dure est fabriqué avec du lait de brebis. Sa consistance et son goût rappellent ceux du fromage italien. "Pecorino \%. La composition de la matière grasse de ce dernier fromage a d'ailleurs fait l'objet d'une étude récente (Del Bianco, 1963).

\section{I. - Méthode expérimentale}

Les acides gras libres à chaîne courte ont été isolés par chromatographie liquide-liquide sur colonne de silicagel et dosés par titrimétrie.

\section{A. - Appareillage.}

L'appareillage utilisé est présenté sur la figure 1. Il est identique à celui décrit par Tchobanov (1961). La colonne, comme le vase de titration, sont isolés de l'air extérieur par une solution alcaline faible; le réservoir, la colonne, ainsi d'ailleurs que l'ampoule à décantation servant à la préparation du mélange des solvants, sont munis de manchons de circulation d'eau.

\section{B. - Mode opératoire.}

a) Systèmes de solvants utilisés.

Trois systèmes de solvants ont été utilisés pour effectuer la chromatographie liquide-liquide. 
Chaque système de solvants est préparé dans une ampoule à décantation munie d'une enveloppe à circulation d'eau $\left(20^{\circ} \mathrm{C}\right)$.

Après agitation énergique et saturation mutuelle des deux couches, le mélange de solvants est laissé au repos jusqu'à nette séparation des phases. Le volume de la phase inférieure (utilisée comme phase stationnaire) peut être évalué à l'aide d'une éprouvette graduée.

Système 1 : éther de pétrole, acétone, eau : 75/15/10

Système 2: (utilisé antérieurement par Tchobanov et Popov) isooctane, acétone, eau : 70/25/5.

Système 3 : isooctane, acétone, eau : 41/52/7.

b) Remplissage de la colonne.

Lors de l'utilisation du système 1 - qui convient pour l'identification des acides de $\mathrm{C}_{2}$ à $\mathrm{C}_{6}+\mathrm{C}_{8}$-, on incorpore $8 \mathrm{~g}$ de silicagel à $9,8 \mathrm{ml}$ de phase stationnaire.

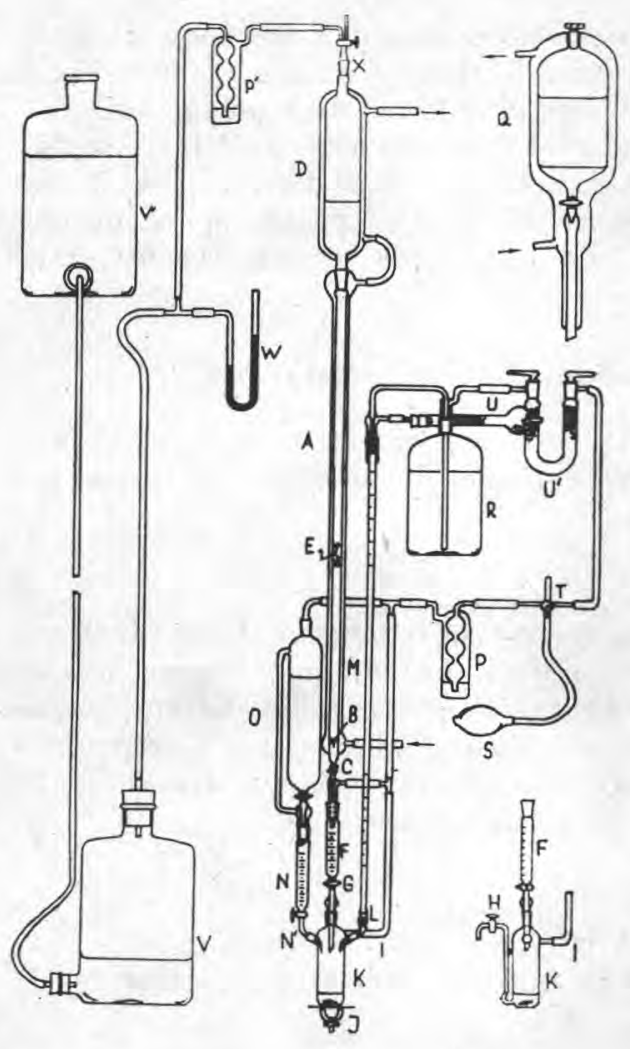

A. Colonne : $40 \mathrm{~cm}$ de long, $1 \mathrm{~cm}$ de diamètre intérieur. - B. Coton ou verre fritté. - D. Réservoir contenant la phase mobile, - E. Rondelles ou fibres de cellulose. - F. Collecteur de fractions. - H. Système d'évacuation du liquide titré. - I. Tube assurant la transmission de la pression. - J. Agitateur magnétique. - K. Vase de titration. - L. Microburette. - NN'. Solution d'indicateur coloré. - O. Réserve d'indicateur. - $\mathrm{PP}^{\prime}$. Régulateur de pression. - Q. Ampoule à décantation munie d'un manchon de circulation d'eau. - R. Réserve de solution de titrage. - S. Poire de caoutchouc créant une pression dans le vase de titration. - T. Robinet à 4 voies. - U. Tubes contenant des fixateurs d'humidité et de $\mathrm{CO}_{2}$. - VV'. Système assurant une pression en D. - W. Niveau de mercure. - X. Communication avec l'extérieur.

\section{Fig. 1.}

Vue d'ensemble de l'appareillage utilisé. 


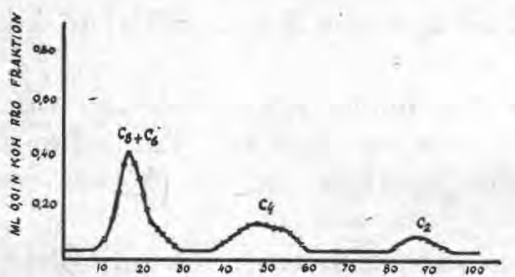

FRAKTIONS ML

Fig. 2. - Chromatogramme de l'éluat obtenu avec le système solvant 1, à partir des acides libres du fromage "Képhalotiri "

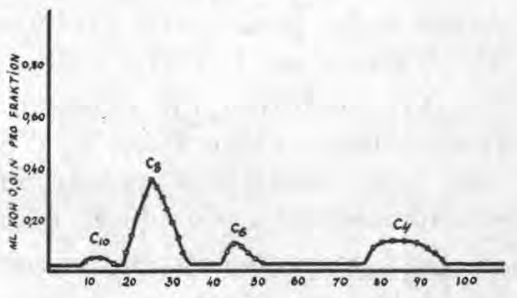

ERAKTIONS ML

Fig. 3. - Chromatogramme de l'éluat obtenu avec les systèmes solvants 2 et 3, à partir des acides libres - du fromage "Képhalotiri "

Lorsqu'on utilise le système 2 (pour l'identification des acides de $\mathrm{C}_{4}$ à $\mathrm{C}_{12}$ par exemple) on incorpore $7 \mathrm{~g}$ de silicagel pour $3,5 \mathrm{ml}$ de phase stationnaire, et on ajoute rapidement $0,33 \mathrm{ml}$ d'une solution aqueuse d'hydroxyde d'ammonium au $1 / 4$.

La préparation du gel et le remplissage de la colonne sont effectués selon la méthode décrite par Zbinowsky (1955).

La hauteur de la colonne de silicagel est voisine de $30 \mathrm{~cm}$. Le débit de l'éluat est de 30 à 40 gouttes par minute.

c) Préparation de l'échantillon de fromage et dépôt sur la colonne.

Un échantillon homogène de fromage est mêlé à un poids égal de sulfate de sodium. A $1 \mathrm{~g}$ du mélange obtenu on ajoute $0,4 \mathrm{ml}$ d'acétone et environ $2 \mathrm{ml}$ de phase mobile. Le tout est mis à la glacière et périodiquement agité. L'ensemble est ensuite versé sur la colonne. Le dépôt de l'échantillon est ainsi effectué de la même façon qu'indiqué ailleurs par Sandev et al. (1963).

d) Titrage des acides.

On recueille l'éluat par fractions de $1 \mathrm{ml}$.

Le titrage est effectué dans un vase isolé de l'air extérieur et sous agitation constante du liquide. Les conditions opératoires sont indiquées sur la figure 1. On utilise une solution N/10 d'hydroxyde de potassium dans l'éthanol, en présence de phénolphtaléine comme indicateur $(0,3$ p. $1000, \mathrm{p} / \mathrm{v})$.

\section{II. - Résultats}

On a pu établir que la reproductibilité de la méthode variait entre 1 à 4 p. 100.

Sur les figures 2 et 3 sont portés les résultats obtenus lors de l'analyse des acides gras libres du fromage "Kephalotiri ».

Le système solvant 1 a été utilisé pour l'obtention de la figure 2 ; les systèmes solvants 2 et 3 ont été utilisés pour l'obtention de la 
figure 3 . Le passage du système 2 au système 3 s'est effectué à la $40^{\mathrm{e}}$ fraction de $1 \mathrm{ml}$ recueillie.

On constate qu'il existe dans les acides gras libres un taux relativement élevé d'acide à 8 atomes de carbone. Par ailleurs, dans les conditions expérimentales décrites, on ne décèle pas d'acides libres à plus de 10 atomes de carbone.

Ceci pourrait s'expliquer soit par la spécificité de décomposition de la matière grasse au cours de la fermentation, soit par le fait que les acides libres comptant plus de 10 atomes de carbone se trouvent, dans le fromage "Kephalotiri ", dans un "état " tel qu'ils ne sont pas élués au cours de la chromatographie.

\section{Résumé}

Une méthode d'identification et de dosage des acides gras libres à bas poids moléculaire est décrite. Cette méthode utilise la chromatographie liquide-liquide sur colonne de silicagel. Elle est appliquée à l'étude des acides gras libres du fromage "Kephalotiri ".

\section{Summary}

A method for identification and dosage of low molecular free fatty acids has been described. This method is based on liquidliquid chromatography on silica gel column and has been applied to the study of the free fatty acis of "Kephalotiri » cheese.

\section{RÉFÉRENCES BIBLIOGRAPHIQUES}

DAy (E. A.), LrbBey (L. M.) 1964. Cheddar cheese flavor : Gas chromatographic and mass spectral analyses of the neutral components of the aroma fraction. J. Food Sci., 29, 583 .

Del Branco (F. M.), 1963. Studio sulla sostanza grassa del formaggio pecorino. Riv. ital. sost. grass, 40, 524.

Gordon (A. H.), Martin (A. J. P.), Synge (R. L. M.), 1943. Biochem. J., 37, 79.

Sandev (S.), Tchobanov (D.), Dardjonov (T.), 1963. Simplified chromatographic determination of steam-volatile fatty acids in rumen fluid. Comptes rendus de l'Acad. bulg. des Sci., 16, 1.

Tсновалоv (D.), 1961. Liquid-liquid chromatography of dicarboxylic acids in presence of pelargonic acid and "azelaìc" glycerids. Comptes rendus de l'Acad. bulg. des Sci., 14, 155.

TсноваNov (D.) et Popov (A.). Communication personnelle.

ZBINOWSKI (V.), 1955. New solvent system for separating monocarboxylic acids $\left(\mathrm{C}_{2}\right.$ to $\left.\mathrm{C}_{16}\right)$ and dicarboxylic acids $\left(\mathrm{C}_{2}\right.$ to $\left.\mathrm{C}_{12}\right)$. Anal. Chem., 27, 764 . 